\title{
HIERARCHICAL HIGHER ORDER CRF FOR THE CLASSIFICATION OF AIRBORNE LIDAR POINT CLOUDS IN URBAN AREAS
}

\author{
J. Niemeyer ${ }^{\mathrm{a}, *}$, F. Rottensteiner ${ }^{\mathrm{a}}$, U. Soergel ${ }^{\mathrm{b}}$, C. Heipke ${ }^{\mathrm{a}}$ \\ ${ }^{\text {a }}$ Institute of Photogrammetry and GeoInformation, Leibniz Universität Hannover, Germany - \\ (niemeyer, rottensteiner, heipke)@ipi.uni-hannover.de \\ ${ }^{\mathrm{b}}$ Institute for Photogrammetry, University of Stuttgart, Germany - \\ uwe.soergel@ifp.uni-stuttgart.de
}

\section{Commission III, WG III/4}

KEY WORDS: Classification, Point Cloud, Higher Order Random Fields, Contextual, Lidar, Urban

\begin{abstract}
:
We propose a novel hierarchical approach for the classification of airborne 3D lidar points. Spatial and semantic context is incorporated via a two-layer Conditional Random Field (CRF). The first layer operates on a point level and utilises higher order cliques. Segments are generated from the labelling obtained in this way. They are the entities of the second layer, which incorporates larger scale context. The classification result of the segments is introduced as an energy term for the next iteration of the point-based layer. This framework iterates and mutually propagates context to improve the classification results. Potentially wrong decisions can be revised at later stages. The output is a labelled point cloud as well as segments roughly corresponding to object instances. Moreover, we present two new contextual features for the segment classification: the distance and the orientation of a segment with respect to the closest road. It is shown that the classification benefits from these features. In our experiments the hierarchical framework improve the overall accuracies by $2.3 \%$ on a point-based level and by $3.0 \%$ on a segment-based level, respectively, compared to a purely point-based classification.
\end{abstract}

\section{INTRODUCTION}

\subsection{Motivation}

The automatic interpretation of airborne lidar point clouds in urban scenes is an ongoing field of research. Usually, one of the first steps for inferring semantics is a point cloud classification. In computer vision and remote sensing it was shown that contextual knowledge can be used to improve classification results (Gould et al., 2008; Schindler, 2012). Conditional Random Fields (CRF) offer a flexible framework for the integration of spatial relations. However, in most related approaches this context is limited to a small local neighbourhood due to computational restrictions. Hence, they do not exploit the full potential of CRF. Operating on segments instead of points is one possibility to integrate more spatial information. Nevertheless, for example Shapovalov et al. (2010) showed that this leads to a loss of $1-3 \%$ in overall accuracy in their experiments compared to a point-based classification due to generalisation errors. A trade-off is the use of a Robust $P^{n}$ Potts model (Kohli et al., 2009), which considers higher order cliques. This model favours the members of a clique to take the same label but also allows for a few members assigned to other classes. This 'soft" segmentation preserves small details in a better way compared to standard segmentation methods. However, interactions between the cliques can not be considered in this model.

The aim of our work is to integrate long range interactions while still preserving small structures in the scene classification. We develop a hierarchical framework consisting of two layers, which considers a supervised contextual classification on a point layer as well as on a segment layer via CRF. In order to avoid generalisation errors, the segments are generated in each iteration based on the results of the point-based classification, and hence they correspond more and more closely to the objects. In this way the advantages of operating on both levels of detail are combined. Our

\footnotetext{
${ }^{*}$ Corresponding author
}

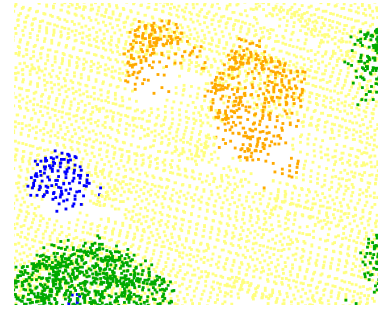

(a) Initial classification

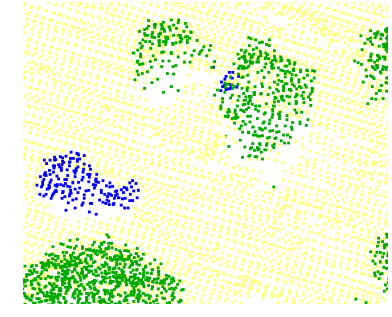

(b) Final classification
Figure 1. Example of a correction of our framework. The two trees in the center of 1(a) are wrongly labelled as building (orange) in the initial point-based classification. After iterating through the two CRF layers several times, these points are correctly classified as tree thanks to context information (1(b)).

iterative procedure enables the correction of potentially wrong decisions at a later stage. Figure 1 shows an example of label hypothesis revision: In a first point-based classification, visualised in Fig. 1(a), the two trees in the centre were wrongly assigned to class building (orange). The integration of context in a larger spatial scale as well as the adoption of the segments in our iterative method lead to an elimination of these errors, and hence to a correct labelling of these points as tree (green) (Fig. 1(b)).

Additionally, we suggest two new contextual features for the classification of airborne lidar point clouds. They describe the relative position and orientation of segments in the scene, and help to further increase the overall accuracy of the classification.

\subsection{Related work}

There are several approaches dealing with combining multiple scales of context. Three main strategies can be identified in the literature: hierarchical frameworks, approaches using higher or- 
der CRF, and the integration of contextual features. Of course, these combinations have also been combined.

The first group are the hierarchical models. Xiong et al. (2011) showed how point-based and region-based classification of lidar data can interact in a pairwise CRF. They proposed a hierarchical sequence of relatively simple classifiers that are applied to segments and points. Starting either with an independent classification of points or segments, in subsequent steps the output of the previous step is used to define context features that help to improve the classification results. In each classification stage, the results of the previous stage are taken as input. This work inspired our methodology. However, we exploit more complex interactions between the objects. Albert et al. (2015) also tackle the challenge of classifying data considering two scales of context. Their application is image classification of land cover (on a superpixel level) and the update of land use objects (on a higher semantical level). Similar to our approach they chose a hierarchical CRF. The major difference is that in our case the object boundaries are not constant, which makes the task more difficult because in addition a good segmentation has to be found.

A further option to consider regional context in the classification process is the use of higher order CRF. We restrict this discussion to the work with point clouds. Kim et al. (2013) introduce a Voxel-CRF framework for jointly estimating the semantic and geometric structure of an indoor RGB-D point cloud for robotic navigation applications. The authors used an associative higherorder model based on Hough voting and categorical object detection with the aim of identifying voxels belonging to the same object. Sengupta and Sturgess (2015) have a similar goal. They performed object detection and reconstruction from stereo images, also operating in voxel space. Both approaches classify voxels of a fixed size. In contrast, we make use of iteratively adapting segments. These segments are generated with the Robust $P^{n}$ Potts model (Kohli et al., 2009), which is more flexible and leads to a 'soft' segmentation. This model favours the members of a clique to be assigned to the same class label. However, members can have a different label. The degree of penalisation depends on the ratio of those members to the total number of clique members. For this reason, it allows for some inhomogeneity, which may preserve small details in the scene. The cliques are generated in advance, for example by a segmentation algorithm. Also multiple segmentations with potentially overlapping segments can be used (Kohli et al., 2009). This enables the consideration of multiple spatial scales. However, interactions between the cliques can not be modelled by Robust $P^{n}$ Potts models; the expressive power is still restricted.

Najafi et al. (2014) set up a non-associative variant of CRF for point clouds. They first performed a segmentation and then applied the CRF to the segments. Overlapping segments in 2D are considered by higher order cliques. The authors additionally modelled height differences with a pattern-based potential. This is useful for terrestrial scans, but in the airborne case the derived features for the vertical are not very expressive due to missing point data for example on façades. Although higher order CRF are becoming more and more important, it is still difficult to to exploit their full potential for the classification of point clouds due to the extensive computational costs. Inference for such models is a challenging task, and until recently only very few nodes could be considered to form a higher order clique for non-associative interaction models, which restricted the applicability of this framework. In the work of Najafi et al. (2014) only up to six segments are combined to one higher order clique to deal with this problem. Pham et al. (2015) proposed the Hierarchical Higher-order Regression Forest Fields. This approach allows to model non-associative relations between cliques by using regression trees. Similar to our framework, a hierarchical approach is used to combine multiple scales of context. Nevertheless, they also use segments of points as their smallest entities, which may introduce generalisation errors.

The third group integrates the long range information into the classification via contextual features. In computer vision the relative location prior (Gould et al., 2008) has become a helpful indicator for the correct classification of image objects. It learns the position of objects with respect to a reference direction in the image. Based on relative location probability maps also relative location features are generated. However, the implementation for a point cloud is more challenging than for terrestrial images because of a missing and well defined reference direction in the scene. Golovinskiy et al. (2009) adapted this idea to terrestrial lidar point clouds. In their work the relative reference for each segment was defined to point into the direction of the closest road. We aim to determine the suitability of a simplified kind of relative position feature for the airborne case of point cloud classification and use the direction to the closest roads as a relative reference for each object, too.

\subsection{Contributions}

In this paper we propose a hierarchical CRF framework. It classifies a point cloud and additionally provides a segmentation. We extend the work of Niemeyer et al. (2015) by applying a higher order Robust $P^{n}$ Potts model to the point-based classification. The segment-based CRF incorporates a larger spatial scale, which helps to support the classification. A new methodology for propagating the information between the layers is presented, which allows the revision of wrong decisions at later stages. Furthermore, and inspired by Gould et al. (2008) and Golovinskiy et al. (2009), two new segment features are proposed which describe the relative arrangement of segments in an airborne point cloud scene. They capture the distance and orientation for each segment with respect to the closest road. Thus, our framework combines the ideas of the three groups presented in Section 1.2.

We begin with a brief introduction in Conditional Random Fields in Section 2, and present our framework in detail in Section 3. Results of our experiments are presented in Section 4. The paper concludes with Section 5.

\section{CONDITIONAL RANDOM FIELDS}

CRF provide a flexible framework for contextual classification. They belong to the family of undirected graphical models. The underlying graph $G(n, e)$ consists of a set of nodes $n$ and a set of edges $e$. We assign a label $y_{i}$ from the set of object classes $L=\left[l_{1}, \ldots, l_{m}\right]$ to each node $n_{i} \in n$ based on observed data $\mathbf{x}$. The vector $\mathbf{y}$ contains the labels $y_{i}$ for all nodes. Each graph edge $e_{i j}$ links two neighbouring nodes $n_{i}, n_{j} \in n$. Thus, it models the relations between pairs of adjacent nodes and represents contextual relations. CRF are discriminative classifiers that model the posterior distribution $p$ directly based on the Gibbs energy $E$ (Kumar and Hebert, 2006)

$$
p(\boldsymbol{y} \mid \mathbf{x}) \propto \exp (-E(\mathbf{x}, \mathbf{y})) .
$$

The most probable label configuration of all nodes is determined simultaneously in the inference step. This is based on minimising the energy given the data by an iterative optimisation process.

In our case the CRF consists of two layers. The actual definitions of the energy functions, graphs, and the terms of the cost function we use are explained in the next section. 


\section{HIERARCHICAL CRF}

We design a hierarchical CRF consisting of two layers in order to realise the iterative classification of points and segments. The goal is to classify the point cloud as accurately as possible. The CRF layout is shown in Fig. 2. First, we perform a point-based classification on the point layer $\left(C R F^{P}\right)$. Subsequently, a second CRF classification operating on segments is applied. This layer is denoted by $C R F^{S}$. The segmentation is obtained by detecting connected components of the same class labels in the classified point cloud. Thus, for each segment also a prior of the semantic label is known which can be used for the extraction of features. In this way the result of $C R F^{P}$ is propagated to $C R F^{S}$. In contrast, the optimisation of $C R F^{S}$ delivers beliefs for each segment to belong to a certain object class. These beliefs are mapped to the point level and incorporated into the next iteration of the $C R F^{P}$ via an additional cost term. In this way the results are propagated along the red dashed lines in Fig. 2 from one step to the next. The basic idea and main motivation of this framework is that some remaining classification errors of $C R F^{P}$ might be eliminated by utilising regional context information between segments instead of points. While local interactions are mainly modelled by the point-based $C R F^{P}$, the segment-based $C R F^{S}$ is able to represent longer range interactions at a regional level. The classification in both layers is applied several times in sequence with the aim of improving the segments iteratively, and transferring regional context to the point-based level. The three components $C R F^{P}$, segmentation, and $C R F^{S}$ are described in the following subsections.

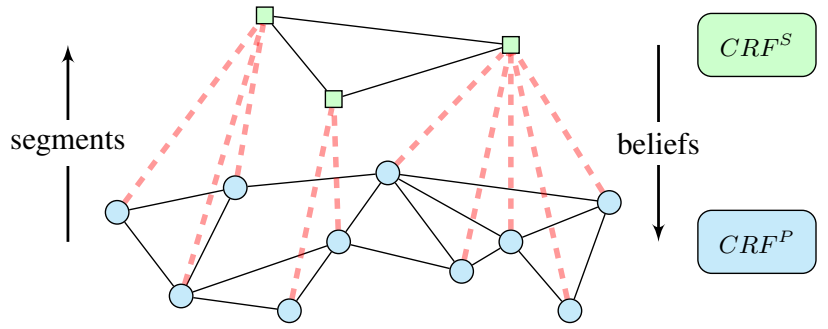

Figure 2. Hierarchical CRF consisting of a point layer (blue) and a segment layer (green). The black edges model the inter-layer relations. The red dashed lines visualise the intra-layer connections, which are considered by defining the segments on the one hand, and by propagating the beliefs of the segment layer to the point layer on the other hand.

\subsection{Point-Based Classification $C R F^{P}$}

We start with the description of the point-based $C R F^{P}$ representing the point layer in our hierarchical framework. Each point represents a node of the graph and is linked by edges to its $k$ nearest neighbours in 2D. This corresponds to a cylindrical neighbourhood which was identified to be more expressive than a spherical neighbourhood. In this case also the larger height differences of the points are modelled, which carry helpful information for distinguishing certain classes (Niemeyer et al., 2011). The energy of $C R F^{P}$ is composed of four terms:

$$
\begin{aligned}
E^{P}(\mathbf{x}, \mathbf{y})= & \sum_{i \in n} E_{u}^{P}\left(\mathbf{x}, y_{i}\right)+\theta_{p}^{P} \sum_{e_{i j} \in e} E_{p}^{P}\left(\mathbf{x}, y_{i}, y_{j}\right) \\
& +\theta_{h}^{P} \sum_{c \in C} E_{h}^{P}\left(\mathbf{x}_{c}\right)+\theta_{\xi}^{P} \sum_{i \in n} E_{\xi}^{P}\left(y_{i}^{S}\right) .
\end{aligned}
$$

The two terms $E_{u}^{P}\left(\mathbf{x}, y_{i}\right)$ and $E_{p}^{P}\left(\mathbf{x}, y_{i}, y_{j}\right)$ in Eq. 2 are the unary and pairwise costs, respectively. $E_{h}^{P}\left(\mathbf{x}_{c}\right)$ models the higher order cost for each clique $c \in C$. The parameters $\theta_{p}^{P}$ and $\theta_{h}^{P}$ act as relative weights of the energy components. A second unary term $E_{\xi}^{P}\left(y_{i}^{s}\right)$ weighted by $\theta_{\xi}^{P}$ is introduced to incorporate the results of the segment-based classification $y_{i}^{S}$. The costs are explained in the following subsections.

3.1.1 Unary Cost The unary cost $E_{u}^{P}\left(\mathbf{x}, y_{i}\right)$ determines the most probable object label of the $m$ classes for a single node given its feature vector $\mathbf{f}_{i}^{P}(\mathbf{x})$. For each node $n_{i}$ such a vector is extracted taking into account not only the data $\mathbf{x}_{i}$ observed at that point, but also at the points in a certain neighbourhood. The cost is modelled to be the negative logarithm of the probability of $y_{i}$ given the data $\mathbf{x}$ :

$$
E_{u}^{P}\left(\mathbf{x}, y_{i}\right)=-\log p\left(y_{i} \mid \mathbf{f}_{i}^{P}(\mathbf{x})\right) .
$$

Any discriminative classifier with a probabilistic output can be used for the computation of the unary cost (Kumar and Hebert, 2006). In this work, we apply a Random Forest (RF) classifier. (Breiman, 2001). It consists of a number $T$ of trees grown in a training step. In order to classify an unknown sample from the dataset, each tree casts a vote for the most likely class based on its features which are presented to the trees. Dividing the sum of all votes for a class by the total number of trees defines a probability measure which is used in Eq. 3 .

3.1.2 Pairwise Cost The term $E_{p}^{P}\left(\boldsymbol{x}, y_{i}, y_{j}\right)$ in Eq. 2 represents the pairwise cost and explicitly incorporates the contextual relations in the classification process. It models the dependencies of a node $n_{i}$ from its adjacent node $n_{j}$ by comparing both node labels and considering the observed data $\mathbf{x}$. We apply a contrastsensitive Potts model (Boykov et al., 2001), which considers the similarity of both adjacent node feature vectors with its Euclidean distance $d_{i j}=\left\|\mathbf{f}_{i}^{P}(\mathbf{x})-\mathbf{f}_{j}^{P}(\mathbf{x})\right\|$ :

$$
E_{p}^{P}\left(\mathbf{x}, y_{i}, y_{j}\right)= \begin{cases}0 & \text { if } y_{i}=y_{j} \\ p_{1}+\left(1-p_{1}\right) e^{-\frac{d_{i j}^{2}}{2 \sigma^{2}}} & \text { if } y_{i} \neq y_{j}\end{cases}
$$

The relative weights of the data-dependent and data-independent smoothing term in Eq. 4 is controlled by the parameter $p_{1} \in$ $[0 ; 1]$. If $p_{1}$ is set to zero, the degree of smoothing depends completely on the data. In contrast, the model becomes a simple Potts model if $p_{1}$ equals one. The parameter $\sigma^{2}$ corresponds to the mean value of the squared feature distances $d_{i j}^{2}$ and is determined during training. The contrast-sensitive Potts model in the point layer classification leads to a data-dependent smoothing effect, preserving small structures with different features.

3.1.3 Higher Order Cost In (Niemeyer et al., 2015) it turned out that many lidar points could not be associated with a segment of $C R F^{S}$ because they were assigned to labels different to their neighbours. Thus, no improvement by the segment-based classification could be obtained for those isolated points. To cope with this problem we additionally make use of the Robust $P^{n}$ Potts model (Kohli et al., 2009) in this study in order to obtain a smoother result. Compared to a standard segmentation, the Robust $P^{n}$ Potts model enables a 'soft' segmentation based on higher order cliques. These cliques are provided in advance, i.e. by a segmentation. In order to distinguish between the segmentation generating the higher order cliques on the one hand, and the segmentation extracting the entities for $C R F^{S}$ (Section 3.2), we denote the former by $\mathrm{Seg}^{P n}$ in this paper. The energy term $E_{h}^{P}\left(\mathbf{x}_{c}\right)$ in Eq. 2 penalises a clique $c$ depending on the amount of members having another label (denoted as $N_{i}\left(\mathbf{x}_{c}\right)$ ), i.e. $N_{i}\left(\mathbf{x}_{c}\right)=\min _{l}\left(|c|-n_{l}\left(\mathbf{x}_{c}\right)\right)$. The amount of variables assigned to class $l$ is given by $n_{l}\left(\mathbf{x}_{c}\right) . Q$ describes the truncation parameter controlling the rigidity of the higher order clique 
cost. The maximum cost $\gamma_{\max }$ is assigned in case of more than $Q$ members with a label different than the dominant label. In case of a more homogeneous labelling of the clique members, the cost is linearly reduced depending on the number of variables not taking the dominant label (Kohli et al., 2009):

$$
E_{h}^{P}\left(\mathbf{x}_{c}\right)= \begin{cases}N_{i}\left(\mathbf{x}_{c}\right) \frac{1}{Q} \gamma_{\max } & \text { if } N_{i}\left(\mathbf{x}_{c}\right) \leq Q \\ \gamma_{\max } & \text { otherwise }\end{cases}
$$

Our goal is to preserve small structures in the point cloud, on the one hand, and to still obtain smooth results serving as input for the segment layer classification $C R F^{S}$, on the other hand. We formulate a cost function depending on the quality and on the size of each clique:

$$
\begin{aligned}
\gamma_{\max } & =\left(\theta_{h p_{1}}^{P}+\theta_{h p_{2}}^{P} \cdot G(\mathbf{x})\right) \cdot|c|^{\theta_{h p_{3}}^{P}} \\
\text { with } G(\mathbf{x}) & =\frac{\left\|\sum_{i \in c} \sum_{l \in L}\left(p_{i, l}\left(y_{i, l} \mid \mathbf{f}_{i}^{P}(\mathbf{x})\right)-\mu_{l}\right)^{2}\right\|}{|c|}
\end{aligned}
$$

The segment quality $G(\mathbf{x})$ is defined by the variance of the posteriors obtained from the unaries (RF classification, Eq. 3) of all points within one clique. In Eq. 7, $\mu_{l}$ represents the mean value of the probabilities belonging to class $l$. A high variance indicates an inhomogeneous clique, leading to a high cost. In contrast, a clique with a dominant label provides a low variance. It represents a good homogeneous clique, and thus its nodes should be favoured to be assigned to the same class. $\theta_{h p_{1}}^{P}$ is a dataindependent smoothing term and $\theta_{h p_{2}}^{P}$ models the relative weight of the data-dependent term. Both parameters are determined in training. $\theta_{h p_{3}}^{P}$ is set manually to control the influence on the clique size. The cliques are constructed with the supervoxelalgorithm, which has been shown to provide good segmentation results for point cloud (Papon et al., 2013). Multiple segmentations can be used for $\mathrm{Seg}^{\mathrm{Pn}}$, and the segments are allowed to overlap. In this way segments of different scales can be considered.

3.1.4 Long Range Cost In order to propagate the information obtained by the larger spatial scale of the segment layer to the next iteration of $C R F^{P}$, an additional energy term $E_{\xi}^{P}\left(y_{i}^{S}\right)$ is introduced:

$$
E_{\xi}^{P}\left(y_{i}^{S}\right)=-\log \left(b e l_{i}^{S}\left(y_{i}^{S}\right)\right)
$$

We map the classification results of $C R F^{S}$ to the point level, where each lidar point $i$ is assigned the beliefs per class of the segment-based classification result. The beliefs are denoted by bel $\left._{i}^{S}\left(y_{i}^{S}\right)\right)$ in Eq. 8. This strategy enables to incorporate the information gained by considering the larger scale into the local point classification.

3.1.5 Features A set of geometrical and waveform based features is extracted for each 3D lidar point representing the feature vector $\mathbf{f}_{i}^{P}(\mathbf{x})$ for each node $n_{i}$. In our case $\mathbf{f}_{i}^{P}(\mathbf{x})$ consists of 12 components. The waveform features are the intensity value as well as the echo ratio, which describes the ratio of the echo number per point and the total number of echoes in the waveform. A larger group of features represents the local geometry of the point cloud. These features are linearity, planarity, scatter, anisotropy, normal of an approximated plane, and the ratio of the sums of the eigenvalues in a $2 D$ and a $3 D$ neighbourhood, which have been shown to lead to good results in (Chehata et al., 2009). The scale of the local neighbourhood for the computation of these features is determined in the way described in Weinmann et al. (2015). Additionally, the feature height above ground is used. It is extracted using the module LASHeight from the software package
LASTools ${ }^{1}$. All features of nodes are scaled to the range $[0,1]$.

3.1.6 Training and inference Our framework is a supervised classification method. Thus, we need a fully labelled reference point cloud for the training of the RF classifier as well as of the weights $\boldsymbol{\Theta}=\left(\theta_{h p_{1}}^{P}, \theta_{h p_{2}}^{P}, \theta_{p}^{P}, \theta_{h}^{P}\right)$.

Learning parameters for higher order random fields is still an active field of research. For our framework we apply a similar heuristic as used by Kohli et al. (2009). Firstly, we learn the relative weight $\theta_{p}^{P}$ between unary and pairwise potentials without considering higher order cliques. Secondly, the higher order parameters $\theta_{h p_{1}}^{P}$ and $\theta_{h p_{2}}^{P}$ are trained without considering pairwise interactions. The last step is to learn the relative weighting $\theta_{h}^{P}$ between the pairwise and the higher order interactions. Training of all parameters simultaneously would lead to very low weights for the higher order costs because both interaction potentials have a similar (smoothing) effect on the result (Kohli et al., 2009). We determine $\Theta$ by maximising the number of correct samples using a part of the training data that was not used for training the energy terms. The relative weight $\theta_{\xi}^{P}$ is set manually, but it can be learned based on training data in the future. The contextual term is not yet available in the initial iteration of $C R F^{P}$. For this reason, the costs are set to be equal for all classes in the first iteration to enable an appropriate training of the relative term weights $\theta_{p}^{P}$ and $\theta_{h}^{P}$.

Inference is the task of finding the optimal label configuration based on minimizing the energy function of Eq. 2 for given parameters. We use the methodology suggested by Kohli et al. (2009), which is able to perform inference on CRF with $P^{n}$ Potts models very efficiently in low polynominal time with a graph-cut based algorithm (Boykov et al., 2001). In order to integrate the additional energy term $E_{\xi}^{P}\left(y_{i}^{S}\right)$ into this framework, we add the contextual costs to the costs of the unary term for each node.

\subsection{Segmentation}

Based on the results of the point-based classification, segments are extracted. For this task, Conditional Euclidean Clustering (Rusu, 2009) is applied as implemented in the Point Cloud Library $^{2}$. It is a variant of a region growing algorithm connecting points which are close to each other and meet additional conditions. In our case the points are allowed to have a distance $d^{\text {seg }}$, and they must have the same label from the point-based classification to be assigned to the same segment. This leads to a segmented point cloud with the advantage of having a prior for the semantic interpretation for each entity, potentially enabling the extraction of more meaningful features for a following segmentbased classification.

\subsection{Segment-Based Classification $C R F^{S}$}

The segments generated in the way described in Section 3.2 are the main entities for $C R F^{S}$ and represent the nodes of the segment layer in our model. Adjacent segments are linked by edges. The adjacency for constructing the graph is given if individual member points of two segments are neighbours in $2 \mathrm{D}$. This is the case if their distance is smaller than a threshold $d_{\text {graph }}^{S}$. The idea of using this second layer is the incorporation of a larger spatial scale. This information is integrated via the additional contextual energy term in Eq. 2 and is assumed to improve the pointbased classification. For this reason the focus of $C R F^{S}$ is not on

\footnotetext{
${ }^{1}$ http://rapidlasso.com/lastools/ (accessed 07/12/2015)

${ }^{2}$ http://pointclouds.org/ (accessed 31/03/2016)
} 
smoothing the labels, but on exploiting context. This CRF utilises only the unary and the pairwise costs with a relative weight $\theta_{p}^{P}$ :

$$
E^{S}(\mathbf{x}, \mathbf{y})=\sum_{i \in n} E_{u}^{S}\left(\mathbf{x}, y_{i}\right)+\theta_{p}^{S} \sum_{i, j \in e} E_{p}^{S}\left(\mathbf{x}, y_{i}, y_{j}\right)
$$

3.3.1 Unary Cost Similar to the point layer, the negative logarithm of the posterior of a segment-based RF classifier is used to define the unary energy $E_{u}^{S}\left(\mathbf{x}, y_{i}\right)$.

3.3.2 Pairwise Cost In contrast to the contrast-sensitive Potts model, a generic model of the local relations between the object classes does not only lead to a smoothing effect but is also able to learn that certain class relations may be more likely than others given the data (Niemeyer et al., 2014). In the segment layer the pairwise costs are modelled by the negative logarithm of the joint posterior probability of two node labels $y_{i}$ and $y_{j}$ given an interaction feature vector $\mathbf{f}_{i j}^{S}(\mathbf{x})$ for each edge $e_{i j}$.

$$
E_{p}^{S}\left(\boldsymbol{x}, y_{i}, y_{j}\right)=-\log p\left(y_{i}, y_{j} \mid \mathbf{f}_{i j}^{S}(\mathbf{x})\right) .
$$

This information is used to improve the quality of classification, with the drawback of having to determine more parameters. We apply another RF classifier to obtain the probabilities for the interactions in a similar way as for the unary costs. The main difference in this case is that $m^{2}$ classes must be distinguished for the pairwise interactions because each object class relation is considered to correspond to a single class.

3.3.3 Features The availability of segments allows for the extraction of a new set of features for nodes and their interactions. We extract the mean and standard deviation of the point intensities as well as of the height above the DTM. Moreover, the maximum difference in elevation is determined. Three features are derived from an approximated plane: the normal direction, the standard deviation of the point normal directions, and the sum of the squared residuals. Additionally, three parameters describe the segment geometry: the area, the volume as well as the point density within the segment. The mean value of the echo ratio helps to detect vegetation. Three more features make use of context: For each segment the most prominent neighbouring class label is determined based on the results of $C R F^{P}$ by counting the labels of direct neighbour segments and using the label with the maximum vote as a feature.

Inspired by the idea of (Golovinskiy et al., 2009), we use two features related to roads which are supposed to describe the relative positions of the segments in the scene. For each segment the distance to a road is computed by searching the closest road points to its centroid. The orientation with respect to the closest road is expressed by the angle between the principal direction and the direction from the segment centroid to the closest road point. The principal direction is obtained by a principal component analysis. In total, 15 features compose the segment feature vectors $\mathbf{f}_{i}^{S}(\mathbf{x})$.

In $C R F^{S}$ all potential class relations are distinguished by the interaction model. For this reason we have to derive discriminative features for each edge. We use the concatenated node feature vectors of both adjacent segments. Additionally, the feature vector is augmented by the differences of intensity, height, and normal direction, the minimum Euclidean distance between the points of two segments, as well as by the mean height differences at the border the segments. This results in an edge feature vector $\mathbf{f}_{i j}^{S}(\mathbf{x})$ consisting of 35 elements.

3.3.4 Training and inference Two independent RF classifiers have to be trained for the unary and pairwise terms, respectively.
In order to learn the interactions of object classes, a fully labelled reference point cloud is needed. The same training areas are used for both layers. Segments are obtained by classifying the training areas and applying the Euclidean clustering in the same way as described before. In order to determine the reference labels for these segments, a majority vote of all point labels contributing to one segment is performed. According to $C R F^{P}$ the relative weight $\theta_{p}^{S}$ is learned by varying the parameter and selecting the value, which leads to the maximum number of correct samples. For inference the max-sum version of the standard message passing algorithm Loopy Belief Propagation (LBP) (Frey and MacKay, 1998) is used. Compared to graph-cuts, LBP has the advantage that it does not require the potentials to be submodular.

\subsection{Alternating optimisation}

The two layers of our framework are optimized independently. We do not model the intra-layer edges which are represented by red dashed lines in Fig. 2 explicitly by edges of a CRF. The reason for that is that a single inference step solving both layers simultaneously is difficult to carry out due to the changing graph structure in $C R F^{S}$. In contrast, information between the layers is propagated in another way with the possibility of enabling an iterative correction of labelling errors. On the one hand, the result of the point-based $C R F^{P}$ is used to generate the segments serving as input for the segment layer $C R F^{S}$. On the other hand, the output of the segment-based classification is propagated into the next iteration of $C R F^{P}$ via the energy term $E_{\xi}^{P}$ (Eq. 8). The computations stop after a manually set number of iterations is reached.

Some kind of smoothing is additionally applied by assigning isolated points not belonging to any segment (and thus remaining unclassified in $C R F^{S}$ ) to the smallest segment in a small spherical neighbourhood of radius $r_{i s o}$. In this way they also receive segment beliefs for the next iteration of $C R F^{P}$. This may serve as an indicator for the isolated points to be classified in the same way as their neighbouring points. The smallest segment in the neighbourhood is chosen for improving the detection of small structures. For instance, it favours an isolated point with a height of $1 \mathrm{~m}$ above the road to belong to the car segment next to it instead of being assigned to the significantly larger road segment.

With regard to the computational effort note that the point features used in $C R F^{P}$ have to be extracted only once. On the other hand the contextual costs and the higher order costs change from iteration to iteration and have to be re-computed. It is also possible to re-use the trained $\mathrm{RF}$ classifier from the first iteration. Considering $C R F^{S}$, the features have to be extracted in every iteration because the segment borders change. Moreover, both RF classifiers (for the unary and the pairwise terms) are trained anew in each iteration. The global weighting parameters $\Theta$ are optimised only once during the first iteration.

\section{EXPERIMENTS}

\subsection{Test setup}

The performance of our method is evaluated on a lidar data set of Hannover, Germany. This scene comprises a densely built-up area consisting of both old and modern buildings as well as a large cemetery with vegetation. It was acquired in March 2010 using a RIEGL LMS-Q560 sensor flying at a mean height of $500 \mathrm{~m}$ above ground. The study area has a size of $258,8 \mathrm{~m}^{2}$ and contains $2,241,111$ points, resulting in a point density of approximately 8.6 points $/ \mathrm{m}^{2}$. Intensities and multiple echoes were recorded. 
A training set consisting of 750,440 points is used for learning the RF classifiers. The relative weights of the energy terms are determined using a second training set comprising 411,114 points. The point cloud which is used for evaluation has $1,079,527$ points. A manual labelling of all points was performed to obtain reference labels for training and accuracy assessment. We distinguish between the six object classes road, natural soil, low vegetation, tree, building, and car in both layers. Note that the different classes do not appear equally often in the data set. In particular, low vegetation and car are rare.

We use three different settings in order to assess the accuracy of our framework and to determine the benefits of the two suggested road features distance and orientation to the closest road for segments:

1. The first classification uses both road features. For their computation the closest segment which was classified as road (and is larger than 10 points for robustness) is detected in order to roughly approximate the road. Thus, it is based on the classification results from $C R F^{P}$. This approach is denoted by $\mathrm{R}_{\text {approx }}$. The parameters are trained using this setting and the results are presented in detail in Section 4.3.

2. In the second classification, denoted as $\mathrm{R}_{O S M}$, an external road data base from OpenStreetMap ${ }^{3}$ is used for the computation of both features. The roads are represented by polylines in this case. The aim is to find out if any improvements can be gained compared to using only approximate roads.

3. For evaluating the impact of both road features, a classification is performed without distance and orientation to the closest road. This result is referred to as $\mathrm{R}_{\text {noRoad }}$.

The classification results of $\mathrm{R}_{O S M}$ and $\mathrm{R}_{\text {noRoad }}$ are compared with $\mathrm{R}_{\text {approx }}$ in Section 4.4.

\subsection{Parameters}

To ensure a fair comparison, the same parameters were used in all experiments. The trained parameters are obtained from $\mathrm{R}_{\text {approx }}$. We performed five iterations of the alternating point- and segmentbased classification in each case. Moreover, the following parameters are used:

Point layer: The graph is constructed with $k=7$ neighbours. $p_{1}$ of the contrast-sensitive Potts model is set to 0.5 as a tradeoff between smoothing and dependence on data. The higher order cliques are constructed from the results of two applications of the supervoxel-algorithm (Papon et al., 2013) $\left(\mathrm{Seg}^{P n}\right)$. We use $0.7 \mathrm{~m}$ and $1.0 \mathrm{~m}$ as different resolutions to obtain segments describing even small objects in a good manner. The number of segmentations as well as their parameters were determined empirically. $Q$ is set to $30 \%, \theta_{\xi}^{P}$ to 0.5 , and $\theta_{h p_{3}}^{P}$ to 0.1 . The weights $\Theta$ for $C R F^{P}$ were determined to be $\theta_{h p_{1}}^{P}=0.7, \theta_{h p_{2}}^{P}=5.84$, $\theta_{p}^{P}=3.55$, and $\theta_{h}^{P}=1.2$ in training. The RF classifier for the unary potential consists of 200 trees, which are trained based on 5000 samples per class.

Segmentation: Points assigned to the same label are connected if they are closer than $d^{\text {seg }}=1 \mathrm{~m}$. The minimum size for each segment is set to three points to allow for an extraction of representative segment features (Section 3.3.3).

Segment layer: In training, $\theta_{p}^{S}$ was determined to be 1.0. The radius for isolated points to be assigned to a segment is $r_{i s o}=$ $1 \mathrm{~m}$. We use 200 trees with 5000 samples per class for the unary costs, and 200 trees with 3000 samples for the interaction RF due to the lower number of available training samples.

\footnotetext{
${ }^{3}$ www.openstreetmap.org/ (accessed 22/02/2016)
}

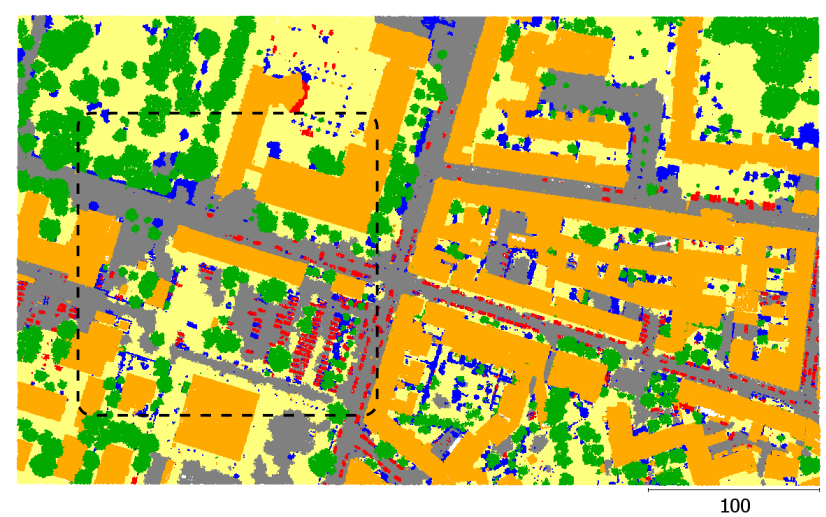

Figure 3. Result of the last $C R F^{P}$ classification for the test area. The colors correspond to the classes road (grey), natural soil (yellow), low vegetation (blue), tree (green), building (orange), and $c a r$ (red). The dashed rectangle illustrates the location of Fig. 4

\subsection{Evaluation}

The result of our classification scheme for $\mathbf{R}_{\text {approx }}$ is visualised in Fig. 3. The corresponding completeness, correctness as well as quality values per class are presented in Tab. 1 for the final $C R F^{P}$ and $C R F^{S}$ results. The quality per class is defined as a measure which takes into account the completeness and the correctness (Heipke et al., 1997). The overall accuracy for the test area is $80.4 \%$ for $C R F^{P}$ and $81.1 \%$ for $C R F^{S}$, which is a reasonable result for the challenging area and the separation of six object classes. The quantitative evaluation in Tab. 1 indicates varying accuracies for the different object classes in terms of completeness and correctness.

The best classification results were obtained for tree and building with high completeness $(>93.5 \%)$ and correctness $(>95.9 \%)$ values, resulting in very good quality values. Thus, these two classes are detected reliably in both classifications. By far the most challenging class is low vegetation in this scenario. It has a poor quality of about $19 \%\left(C R F^{P}\right)$ and $25 \%\left(C R F^{S}\right)$ due to low correctness values. Confusion mainly occurs with the classes natural soil, building, and tree. Apparently the class of low vegetation is not well defined and the features are not expressive enough to distinguish the objects correctly. In particular, objects of low vegetation show various appearances in the data. In general, every class is slightly improved in quality by applying the segment-based classification.

Table 1 additionally provides the difference to the initial pointbased classification $C R F^{P}$ in parenthesis. Positive values (in green) correspond to a better result of our iterative framework. After the first run of $C R F^{P}$ an overall accuracy of $78.1 \%$ is achieved, which can be used for comparison because no interaction with the segment layer took place. This result is improved by alternating through the two layers of our framework. The information mutually propagated between point and segment-based levels increases the overall accuracy by $2.3 \%$ to $80.4 \%\left(C R F^{P}\right)$ and by $3.0 \%$ to $81.1 \%\left(C R F^{S}\right)$ after five iterations.

Compared to the first result only the quality of tree is reduced by $0.9 \%$ for $C R F^{P}$ (see negative values in red), whereas it is increased in $C R F^{S}$ by the same magnitude. For the remaining object classes an improvement of the quality values can be observed. In particular, the class car benefits significantly from our framework. In both cases the quality is raised by almost $28 \%$ mainly 


\begin{tabular}{|c|c|c|c|c|c|c|c|c|c|c|c|c|}
\hline \multirow{3}{*}{$\begin{array}{l}\text { Class } \\
\text { Road }\end{array}$} & \multicolumn{6}{|c|}{$C R F^{P}$} & \multicolumn{6}{|c|}{$C R F^{S}$} \\
\hline & \multicolumn{2}{|c|}{ Completeness } & \multicolumn{2}{|c|}{ Correctness } & \multicolumn{2}{|c|}{ Quality } & \multicolumn{2}{|c|}{ Completeness } & \multicolumn{2}{|c|}{ Correctness } & \multicolumn{2}{|c|}{ Quality } \\
\hline & 58.9 & $(+4.0)$ & 65.7 & $(+8.2)$ & 45.0 & $(+6.0)$ & 58.9 & $(+4.0)$ & 65.7 & $(+8.2)$ & 45.1 & $(+6.0)$ \\
\hline Natural Soil & 75.5 & $(+3$. & 69.9 & $(+4$ & 56.9 & & 76.3 & & 69.8 & & 57.3 & \\
\hline LowVeg & 41.7 & $(+15$. & 26.0 & $(-1$ & 19 & & 46.1 & $(+19$ & 34.8 & $(+7$ & 24.7 & $(+9.0)$ \\
\hline & 93.5 & $(+0.1)$ & 95.6 & $(-1.2)$ & 89.6 & $(-0.9$ & 95.1 & $(+1$. & 95.9 & $(-0$. & 91.3 & $(+0.8)$ \\
\hline Buildin & 95.0 & $(-0.8)$ & 97.6 & $(+1.1)$ & 92.8 & $(+0.3$ & 95.5 & $(-0.3)$ & 97.1 & $(+0.7)$ & 92.9 & $(+0.4)$ \\
\hline Car & 52.3 & $(+34.7)$ & 73.7 & $(+2.8)$ & 44.1 & $(+27.6)$ & 47.7 & $(+30.1)$ & 86.4 & $(+15.4)$ & 44.4 & $(+27.9)$ \\
\hline
\end{tabular}

Table 1. Completeness, correctness and quality in [\%] after the last iteration of $C R F^{P}$ and $C R F^{S}$, respectively. The comparison to the first iteration of $C R F^{P}$ (without any information obtained by segments) is shown in parenthesis. Values in green indicate the improvements of our alternating framework. The overall accuracy is $80.4 \%(+2.3)$ in case of $C R F^{P}$ and $81.1 \%(+3.0)$ for $C R F^{S}$.

due to improved completeness values. This can be explained by the fact that some object points were lost due to smoothing in the initial classification because of the Robust $P^{n}$ Potts model. Also road, natural soild and low vegetation can be detected more accurately in the point and in the segment layer. These classes benefit from the propagated context information. Some errors introduced in the first point-based classification can be corrected in this way. One example is the relatively challenging separation between the classes road and natural soil in the data set. The most important feature for their separation is the intensity value of the lidar points. However, there are many macadam roads, parking areas, and pathways (at the cemetery in the upper left part of Fig. 3) which tend to show ambiguous appearance. In some cases our framework is able to correct the initially assigned wrong labels because the shape and the position of the segment are not very likely.

A further advantage of our methodology is the information about segments. In addition to the low level point cloud classification, we obtain segments roughly corresponding to instances of objects, and we also know their class label. This allows us to estimate the number of object instances for each class in the scene. For example, we detected 512 car segments in the scene. Figure 4 shows some tree segments on the left hand side as well as individual car segments on the right hand side. Due to the lack of reference data for these objects, we can not perform an qualitative evaluation.

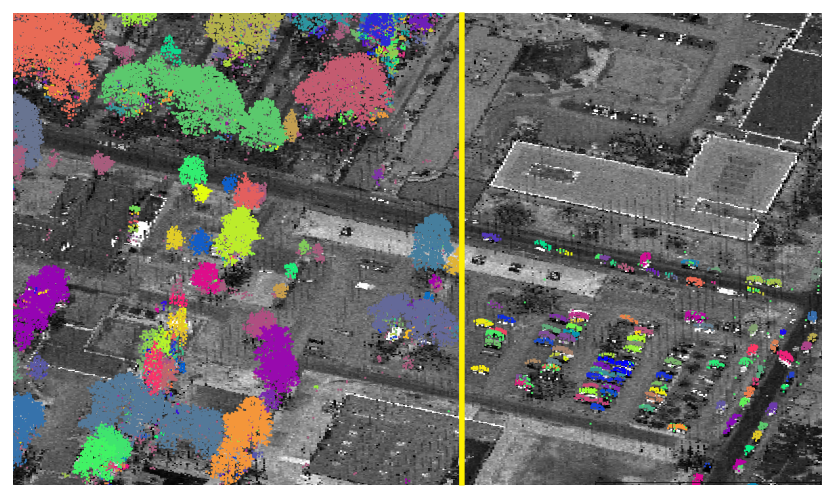

Figure 4. In addition to the point cloud labels, also individual segments approximating the object instances in the scene are derived for each class. The left part of this $3 \mathrm{D}$ view shows the tree segments, on the right side individual cars are represented by segments. A greyscale intensity image of the scene is visualised in the background. The location of the scene is illustrated by the dashed rectangle in Fig. 3.

\subsection{Road features}

This investigation analyses the influence of the two segment features distance and orientation to the closest road. The computa-

\begin{tabular}{l|cc|cc}
\hline \multirow{2}{*}{ Class } & \multicolumn{2}{|c|}{$C R F^{P}$} & \multicolumn{2}{c}{$C R F^{S}$} \\
& $\mathrm{R}_{\text {approx }}$ & $\mathrm{R}_{O S M}$ & $\mathrm{R}_{\text {approx }}$ & $\mathrm{R}_{\text {OSM }}$ \\
\hline Road & +3.3 & +2.5 & +4.0 & +3.2 \\
Natural Soil & +3.0 & +3.3 & -1.4 & -1.2 \\
Low Veg. & -2.5 & -0.4 & -2.4 & -5.0 \\
Tree & +1.3 & +0.2 & +0.9 & +0.1 \\
Building & +0.6 & 0.0 & +0.1 & -1.0 \\
Car & -4.7 & +2.0 & -7.5 & +0.8 \\
\hline Overall Accuracy & +1.5 & +1.3 & +0.2 & -0.1 \\
\hline
\end{tabular}

Table 2. Comparison of quality values and overall accuracies [\%] with respect to $\mathrm{R}_{\text {noRoad }}$ to determine the influence of the features distance to road and orientation to road.

tion of these features in $\mathrm{R}_{\text {approx }}$ (Section 4.3) was based on an approximation of the road. In this section a comparison to $\mathrm{R}_{O S M}$ and $\mathbf{R}_{\text {noRoad }}$ as described in Section 4.1 is carried out.

The results are summarised in Tab. 2 by presenting the differences in quality and overall accuracy with respect to $\mathrm{R}_{\text {noRoad }}$ after the last $C R F^{P}$ and $C R F^{S}$ classification, respectively. Negative values (in red) indicate a better accuracy of $\mathrm{R}_{\text {noRoad }}$. The features are used at the segment layer and the information is then propagated to the point layer iteration by iteration. It becomes evident that the two introduced road features distance and orientation of segments to roads improve the results for the (final) point layer. The overall accuracies increase by $1.5 \%\left(\mathrm{R}_{\text {approx }}\right)$ and $1.3 \%\left(\mathrm{R}_{O S M}\right)$, respectively. For $C R F^{S}$ the impact is only marginal.

Considering the quality values it turns out that 14 of the 24 indicators are improved by the new features. In contrast, the quality decreases in 9 cases. In one case no difference was detected. The class road itself benefits most from the road information. The quality increases by $>2.5 \%$ on the point level and by $>3.2 \%$ on the segment level. Tree is also affected positively. It becomes apparent that the less prominent class low vegetation suffers from the incorporation of these features. Due to the varying shape of low vegetation no representative orientation to the road can be learned. Additionally, the principal direction can not be computed robustly for small segments consisting of only a few points. A similar behaviour is observed for car: the quality is reduced only in case of the approximated road. Apparently, some isolated segments classified as road on the parking areas distort the features of some cars in $\mathrm{R}_{\text {approx }}$. On the other hand, the detection of cars is improved if the OSM data are used, which only contain the skeletons of the roads. Parking areas are not considered in this case, and hence all cars located in this area have more homogeneous features concerning their orientation and distance to the road polyline.

We conclude that our hierarchical framework consisting of $C R F^{P}$ and $C R F^{S}$ allows for the utilisation of road information, which improves the point-based overall accuracy by more than $1.3 \%$. The experiments on this dataset show a comparable performance 
for the approximated road and the external road database. However, expressive segments are necessary in order to describe the object orientation in an appropriate way. Further investigations will show if these features can be used to improve the detection rate of small classes, too.

\section{CONCLUSION AND OUTLOOK}

We have proposed a hierarchical classification framework for airborne lidar point clouds based on a two layer Conditional Random Field (CRF). Both layers are applied in sequence and interact mutually. This approach is able to incorporate local and regional context. In our experiments an increase of $2.3 \%$ (pointbased) and $3.0 \%$ (segment-based) in overall accuracy is observed. We could demonstrate that the suggested iterative framework improves the classification accuracy and allows for the correction of errors in the previous results at a later stage. In particular, the class car benefits from our methodology. Moreover, we introduced two new segment features for airborne point clouds describing the distance and the orientation of each segment with respect to the closest road. These features are helpful and improve the overall accuracy by up to $1.5 \%$ in our experiments.

In future work we intend to improve the higher order cost function $\gamma_{\max }$ as well as the road features for a better detection of under-represented object classes. Furthermore, we will evaluate the performance of our approach using more test areas with different characteristics, and investigate the required amount of training data.

\section{REFERENCES}

Albert, L., Rottensteiner, F. and Heipke, C., 2015. An iterative inference procedure applying Conditional Random Fields for simultaneous classification of land cover and land use. In: ISPRS Annals of Photogrammetry, Remote Sensing and Spatial Information Sciences, Vol. II-3/W5, pp. 1-8.

Boykov, Y., Veksler, O. and Zabih, R., 2001. Fast approximate energy minimization via graph cuts. IEEE Transactions on Pattern Analysis and Machine Intelligence 23(11), pp. 1222-1239.

Breiman, L., 2001. Random Forests. Machine learning 45(1), pp. 5-32.

Chehata, N., Guo, L. and Mallet, C., 2009. Airborne Lidar Feature Selection for Urban Classification using Random Forests. In: International Archives of Photogrammetry, Remote Sensing and Spatial Information Sciences, Vol. XXXVIIInumber Part3-W8, ISPRS, Paris, France, pp. 207-212.

Frey, B. and MacKay, D. J. C., 1998. A revolution: Belief propagation in graphs with cycles. In: Advances in neural information processing systems, Vol. 10, The MIT Press, pp. 479-485.

Golovinskiy, A., Kim, V. G. and Funkhouser, T., 2009. Shapebased recognition of 3D point clouds in urban environments. In: Proceedings of the IEEE International Conference on Computer Vision, pp. 2154-2161.

Gould, S., Rodgers, J., Cohen, D., Elidan, G. and Koller, D., 2008. Multi-class segmentation with relative location prior. International Journal of Computer Vision 80(3), pp. 300-316.

Heipke, C., Mayer, H., Wiedemann, C. and Jamet, O., 1997. Evaluation of Automatic Road Extraction. In: International Archives of Photogrammetry and Remote Sensing, Vol. 32(34W2), pp. 151-156.
Kim, B.-S., Kohli, P. and Savarese, S., 2013. 3D scene understanding by Voxel-CRF. In: Proceedings of the IEEE International Conference on Computer Vision, pp. 1425-1432.

Kohli, P., Ladický, L. L. and Torr, P. H. S., 2009. Robust higher order potentials for enforcing label consistency. International Journal of Computer Vision 82(3), pp. 302-324.

Kumar, S. and Hebert, M., 2006. Discriminative Random Fields. International Journal of Computer Vision 68(2), pp. 179-201.

Najafi, M., Namin, S. T., Salzmann, M. and Petersson, L., 2014. Non-associative higher-order markov networks for point cloud classification. In: Proceedings of the European Conference on Computer Vision, Springer, Zurich, pp. 500-515.

Niemeyer, J., Rottensteiner, F. and Soergel, U., 2014. Contextual classification of lidar data and building object detection in urban areas. ISPRS Journal of Photogrammetry and Remote Sensing 87, pp. 152-165.

Niemeyer, J., Rottensteiner, F., Soergel, U. and Heipke, C., 2015. Contextual classification of point clouds using a two-stage CRF. International Archives of the Photogrammetry, Remote Sensing and Spatial Information Sciences XL-3/W2, pp. 141-148.

Niemeyer, J., Wegner, J. D., Mallet, C., Rottensteiner, F. and Soergel, U., 2011. Conditional Random Fields for urban scene classification with full waveform LiDAR data. In: Photogrammetric Image Analysis, Lecture Notes in Computer Science, Vol. 6952 , Springer, Munich, Germany, pp. 233-244.

Papon, J., Abramov, A., Schoeler, M. and Worgotter, F., 2013. Voxel cloud connectivity segmentation - Supervoxels for point clouds. In: Proceedings of the IEEE Conference on Computer Vision and Pattern Recognition, pp. 2027-2034.

Pham, T. T., Reid, I., Latif, Y. and Gould, S., 2015. Hierarchical Higher-order Regression Forest Fields : An application to 3D indoor scene labelling. In: Proceedings of the IEEE International Conference on Computer Vision, pp. 2246-2254.

Rusu, R. B., 2009. Semantic 3D object maps for everyday manipulation in human living environments. $\mathrm{PhD}$ thesis, Technische Universität München, Germany.

Schindler, K., 2012. An overview and comparison of smooth labeling methods for land-cover classification. IEEE Transactions on Geoscience and Remote Sensing 50(11), pp. 4534-4545.

Sengupta, S. and Sturgess, P., 2015. Semantic Octree : Unifying recognition, reconstruction and representation via an octree constrained higher order MRF. In: IEEE International Conference on Robotics and Automation, pp. 1874-1879.

Shapovalov, R., Velizhev, A. and Barinova, O., 2010. Nonassociative markov networks for 3D point cloud classification. In: International Archives of Photogrammetry, Remote Sensing and Spatial Information Sciences, Vol. 38number 3A, pp. 103-108.

Weinmann, M., Jutzi, B., Hinz, S. and Mallet, C., 2015. Semantic point cloud interpretation based on optimal neighborhoods, relevant features and efficient classifiers. ISPRS Journal of Photogrammetry and Remote Sensing 105, pp. 286-304.

Xiong, X., Munoz, D., Bagnell, J. A. and Hebert, M., 2011. 3-D scene analysis via sequenced predictions over points and regions. In: IEEE International Conference on Robotics and Automation, pp. 2609-2616. 